\title{
Is Intravenous Iron Treatment a Risk for Infection?
}

\author{
María José García ${ }^{1}$, Carmen Alonso ${ }^{1}$, Manuel Fernandez-Miera ${ }^{2}$, Maite Arias ${ }^{1}$, Paula Iruzubieta ${ }^{1}$, Carmen Fariñas ${ }^{3}$, Javier Crespo ${ }^{1}$ \\ and Emilio Fábrega*1 \\ ${ }^{1}$ Gastroenterology and Hepatology Unit, University Hospital "Marqués de Valdecilla", Instituto de Investigación Marqués de Valdecilla (IDIVAL), \\ Santander, Spain \\ 2Department of Internal Medicine, University Hospital "Marqués de Valdecilla", Santander, Spain \\ ${ }^{3}$ Infectious Diseases Unit, University Hospital "Marqués de Valdecilla", Instituto de Investigación Marqués de Valdecilla (IDIVAL), Santander, Spain
}

${ }^{*}$ Correspondence to: Emilio Fábrega, Gastroenterology and Hepatology Unit, University Hospital "Marqués de Valdecilla", Avenida Valdecilla s/n. Santander 39008, Cantabria, Spain; Tel: 073442202544; FAX number: 073442202544.

Received: March 03, 2017; Accepted: March 15, 2017; Published: March 19, 2017;

\begin{abstract}
Background: Iron is a pro-oxidant and an essential nutrient for different bacteria. Experimental animal studies have demonstrated exacerbating sepsis episodes. Intravenous iron treatment has been implicated, at least theoretically in increasing infection episodes. However, these studies did not obtain any conclusion in this respect. Therefore, the aim of our study was to evaluate whether there is a risk between intravenous iron treatment and infection episodes following iron treatment.
\end{abstract}

Methods: In our study, 186 patients who received intravenous iron treatment were included. The biochemical parameters included "pre and post iron treatment" (e.g., ferritin, serum iron, transferring saturation, and hemoglobin) and the later emergence of infections. Moreover, we evaluated all possible risk factors that could interfere with the subsequence appearance of infection.

Results: Infectious complications were reported in 24 patients (12.8\%) after the iron treatments began, and no infection was reported in $87.15 \%(\mathrm{n}=162)$ of the patients. The most common infections were urinary (33\%) and respiratory infections (25\%). The most common infectious agents were enterobacteriae (e.g., E coli, E faecalis, and E faecium). Notably, we did not observe any intracellular pathogens. In our statistical survey, we did not find a relationship between infection onset and intravenous iron treatment. To analyze the baseline disease, comorbidities, such as diabetes and other treatment (e.g., corticosteroids or anti-TNF) were evaluated, and, no association was found.

Conclusion: In our study, intravenous iron was an effective treatment to correct anemia, and it did not appear to correlate with the development of secondary infections.

Key words: iron therapy; intravenous iron; iron infection.

Abbreviations: IV - intravenous; HD - hemodialysis.

\section{Introduction}

Iron is an important nutrient for many bacteria, and in laboratory animals, it was shown to exacerbate sepsis. [1] It has been postulated that increased plasma iron is responsible for the association of hemochromatosis with infections by Vibrio vulnificus, Yersinia enterocolitica, and Escherichia coli. [2] Accordingly, there are host defense mechanisms that tend to restrict the available iron from invading pathogens. [3] For example, the transferrin receptor is the major pathway for delivery of iron to peripheral tissues through endocytosis of its ligand, transferrin, which binds to iron and circulates in the plasma. Down regulation of the transferrin receptor limits the cell's ability to acquire transferrin-bound iron and concomitantly reduces the endosomal pool of iron that can be accessed by intracellular pathogens, such as L. pneumophila, Mycobacterium tuberculosis, and Mycobacterium avium, and thus restricts their growth. [4, 5] Iron repletion with oral iron has been associated with adverse outcomes for certain infections. In a study of 137 iron-deficient Somali nomads, who were treated with placebo or oral iron (71 subjects), Murray et al. noted seven infection episodes in the placebo group and 36 in the oral iron group (including reactivation of preexisting malaria, brucellosis, and tuberculosis). [6] A large iron supplementation trial of young children on Pemba Island, was prematurely terminated as a result of an excess of serious adverse events (including deaths) in those receiving iron. [7] Ferric iron (Fe)-carbohydrate complexes are widely used for treating Fe deficiency in patients who are unable to meet their $\mathrm{Fe}$ requirements with oral supplements. [8] Intravenous (IV) $\mathrm{Fe}$ is generally well tolerated and effective in correcting Fedeficient states. However, complexing Fe to carbohydrate polymers does not block its potent pro-oxidant effects; therefore, systemic free radical generation and, possibly, tissue damage may result. [9] On the other hand, excessive iron administration may lead to oversaturation of transferrin and the release of free, catalytically active iron into circulation (NTBI) [10] which could be readily utilized by bacteria, leading to their multiplication and the possibility of an overwhelming infection. $[11,12]$ The aim of the present work was to determine the short-term infection rate associated with IV iron treatment and the 
possible risk factors that can predict these infections in a contemporary patient cohort.

\section{Material and methods}

Patient enrollment. A retrospective, longitudinal study was carried out at Marqués de Valdecilla University Hospital (Santander, Spain). The hospital records of patients who underwent ambulatory treatment with IV iron at the hospital day center between January 2011 and December 2012 were reviewed.

Patient parameter evaluation. The medical records of these patients were searched after institutional review board approval was obtained for the following data fields: a) demographic features; b) principal diagnosis; c) comorbid factors, such as diabetes mellitus, arterial hypertension, and dislipemia; d) concomitant treatment as potential infection causes, such as corticosteroids, immunosuppresive therapy, and anti-TNF treatment; e) previous infection, and; f) laboratory data prior to and after IV iron treatment, such as hemoglobin concentration, mean cell hemoglobin $(\mathrm{MCH})$, mean cell hemoglobin concentration (MCHC), red cell volume distribution width (RDW), serum iron, transferrin saturation, and serum ferritin.

Intravenous iron treatment. One thousand $\mathrm{mg}(50 \mathrm{mg} / \mathrm{ml})$ of ferric carboxymaltose was administered in an ambulatory regimen to the subjects at the hospital day center. The doses of this treatment were adapted subsequently according to the Ganzoni equation and their efficacy.

Case definitions. We used a retrospective cohort design with a 1-month baseline period. After IV iron treatment was started, all of the patients were followed for 3 months. We looked for any infectious diseases, and we differentiated the subjects by bacterial, viral and fungal infections, as well as by gastrointestinal, genitourinary, respiratory and other diseases. If a single blood culture was positive for coagulase-negative staphylococci, Corynebacteria, or Bacillus sp., the culture was considered contaminated or representative of a transient bacteremia but not of a bacteremic episode. All other situations in patients in whom at least one blood culture was positive were defined as bacteremic episodes.

Statistical Analysis. The results were analyzed with the SPSS 15.0 computer software package (Statistical Package for Social Sciences, Inc., Chicago. IL). Continuous variables were summarized as the means or as the medians and ranges. Categorical variables were compared with the chi-square and Fisher's exact tests. Correlations between the data sets were examined using the Pearson (r) or Spearman rank (rs) correlation coefficients. Any differences were considered significant for all statistical tests at $\mathrm{p}$ values of less than 0.05 .

\section{Results}

A total of 186 adult patients (70 men and 116 women; mean age, 63 yrs; range, 18 to 80 yrs) were enrolled. The principal diagnosis of the patients included in the study were cardiac diseases $(n=41$, $22 \%)$, neoplasm diseases $(n=32,17.2 \%)$, renal diseases $(n=31$, $16.7 \%)$, malnutrition due to bariatric surgery $(n=19,10.2 \%)$, hepatic diseases $(n=15,8.1 \%)$, and lung diseases $(n=15,8.1 \%)$ (Table 1). A comorbid condition known to increase the infection risk was found in 88 patients $(47.3 \%)$, which included diabetes mellitus $(\mathrm{n}=53)$, current malignancy $(\mathrm{n}=32)$ (under treatment and/or not cured), being a transplant recipient $(n=3)$, and having both a malignancy and diabetes mellitus $(n=5)$. Twelve patients were treated with corticosteroids and six were treated with anti-TNF therapy. The pretreatment median hemoglobin level was $9.5 \mathrm{gr} / \mathrm{dL}$ (5.6- $12.6 \mathrm{gr} /$ $\mathrm{dL}$ ) and after the treatment it was $11.8 \mathrm{gr} / \mathrm{dL}$ (7.4-14.1 gr/dL) (Table 2). Additionally, we found a statistically significant difference between the baseline biochemical iron metabolism parameters pre-and post IV iron treatment (Table 2). Thus, we corroborated that IV iron was an effective therapy in our patients.

Table 1. The principal diagnosis of the patients included in the study.

\begin{tabular}{|l|l|}
\hline \multicolumn{1}{|c|}{ Disease } & \multicolumn{1}{c|}{ n (\%) } \\
\hline Cardiac diseases & $41(22 \%)$ \\
\hline Neoplasms diseases & $32(17.2 \%)$ \\
\hline Kidney disease & $31(16.7 \%)$ \\
\hline Bariatric surgery & $19(10.2 \%)$ \\
\hline Liver diseases & $15(8.1 \%)$ \\
\hline Lung diseases & $15(8.1 \%)$ \\
\hline Others & $33(17.7 \%)$ \\
\hline
\end{tabular}

Table 2. The baseline laboratory iron metabolism parameters pre-and post-intravenous iron treatment.

\begin{tabular}{|l|c|c|c|}
\hline & Pre-treatment & Post-treatment & P \\
\hline Hemoglobin (gr/dL) & $9.5(5.6-14.5)$ & $11.8(7.4-14.10)$ & $<0.01$ \\
\hline MCH (pg/RBC) & $26.4(8.6-37.5)$ & $29.9(21.8-37.4)$ & $<0.01$ \\
\hline MCHC (gr/dL) & $32.4(28.6-35.3)$ & $33.1(25.5-35.3)$ & $<0.01$ \\
\hline RDW (\%) & $18.9(12.2-37.4)$ & $21.4(9-43.6)$ & $<0,01$ \\
\hline Serum iron (mcg/dL) & $25(10-96)$ & $69(16-177)$ & $<0.541$ \\
\hline $\begin{array}{l}\text { Transferrin saturation } \\
\text { (Fe/TIBC)\% }\end{array}$ & $7(2-37)$ & $23,5(5-62)$ & $<0.01$ \\
\hline Serum ferritin & $54(2-753)$ & $408(10-1492)$ & $<0.01$ \\
\hline
\end{tabular}

$\mathrm{MCH}$ - mean cell hemoglobin;

MCHC - mean cell hemoglobin concentration;

RDW - red cell volume distribution width.

No patients had more than one bacteremic episode within the month prior to treatment. Twenty-four patients (12.9\%) suffered from one infection episode after the IV iron treatment, and 162 patients $(87.1 \%)$ did not have any infection episodes. The majority of these infections were from bacterial microorganisms $(75 \%, \mathrm{n}=18)$ followed by unknown etiologies $(16.7 \% \mathrm{n}=4)$ and viral episodes $(8.3 \%, \mathrm{n}=2)$. There were no fungal infections. The urinary tract was involved in $33.3 \%$ of the cases $(n=8) ; 25 \%$ of the cases involved therespiratory system $(n=6)$, which was similar to the unknown focus case percentage; and $16.7 \%$ of the cases involved the gastrointestinal system $(n=4)$. The infectious agent was unknown in $63.5 \%$ of the cases $(n=15)$. The pathogens responsible for these episodes were E coli $(16.7 \%, n=4)$ followed by E faecalis, E faecium, Lysteria and Proteus mirabilis, which all occurred in $4.2 \%$ of the cases $(n=1)$. No intracellular microorganisms or non-capsulated bacteria were found in our series. Additionally, no serious diseases and no mortality cases were reported three months after the treatments began. 
The main characteristics of the patients with and without infection episodes are shown in Table 3. More patients with an infection episode had a comorbidity (diabetes or malignancy) and ongoing immunosuppressive therapy than the patients without infections. However, this was not statistically significant. Furthermore, when we analyzed the relationship between infections with other comorbidities, such as diabetes, cancer, transplantation or treatments such as steroids or anti-TNF we did not find any association.

Table 3. Comorbidity incidences in the infection or no infection groups.

\begin{tabular}{|l|l|l|l|}
\hline & \multicolumn{1}{|c|}{ Infection } & \multicolumn{1}{|c|}{ No Infection } & \multicolumn{1}{c|}{ p } \\
\hline Diabetes mellitus & $8(33.3 \%)$ & $45(28.3 \%)$ & NS \\
\hline Arterial hypertension & $14(58.3 \%)$ & $65(40.1 \%)$ & NS \\
\hline Dislipemia & $5(20.8 \%)$ & $23(14.2 \%)$ & NS \\
\hline Cardiac diseases & $7(29.2 \%)$ & $34(21 \%)$ & NS \\
\hline Renal diseases & $7(29.2 \%)$ & $24(14.8 \%)$ & NS \\
\hline Lung diseases & $1(4.2 \%)$ & $14(8.6 \%)$ & NS \\
\hline Liver diseases & $1(4.2 \%)$ & $14(8.5 \%)$ & NS \\
\hline Bariatric surgery & $3(12.5 \%)$ & $16(9.9 \%)$ & NS \\
\hline Neoplasm & $7(29.2 \%)$ & $25(15.4 \%)$ & NS \\
\hline Organ transplantation & $0(0 \%)$ & $3(1.9 \%)$ & NS \\
\hline Corticosteoids & $3(12.5 \%)$ & $9(5.6 \%)$ & NS \\
\hline Biologic therapy & $1(4.2 \%)$ & $5(3.1 \%)$ & NS \\
\hline
\end{tabular}

NS - Non-Significant

\section{Discussion}

Almost all life forms require iron because of its involvement in basic cellular processes. Free iron was shown to potentiate bacterial growth in vitro. [13] In fact, during infections, pathogens use various means to acquire iron from their hosts, whereas hosts attempt to withhold it from pathogens. [14, 15] Iron therefore represents a point of conflict between the host and the pathogen, and an altered iron balance associated with poor outcomes in several infectious diseases, including malaria, [7] tuberculosis, [16] and HIV-1 infection. [17] Furthermore, certain bacterial species, including E. Coli, Klebsiella spp., and Salmonella spp., use unbound iron in the blood to enhance their growth. These organisms release siderophores, which are iron chelators, into the blood. Once these siderophores are released, bacteria can compete with proteins, such as transferrin for unbound iron in the serum. [18, 19] Staphylococcus aureus and Haemophilus influenzae do not possess siderophore; however, they do have transferrin receptors, which allow these bacteria to use iron for growth. [18] Vibrio vulnificus cannot grow if there is no iron available, and their virulence depends on the ability to obtain iron combined with transferrin. Hepcidin, which is produced during innate immune responses to infections, reduces the iron availability in the serum by inhibiting ferroportin function in macrophages and enterocytes and potentially limits extracellular pathogen growth therein. [20] Intravenous iron may also impair immune function and increase infection susceptibility. [21] Gupta et al. [22] found that exposure of mononuclear cells to IV iron agents induced significant intracellular oxidative stress and shortened CD4+ T lymphocyte survival. High doses of IV iron agents impaired the phagocytic activity and microbial killing capability of polymorphonuclear leukocytes.
[23-25] Furthermore, in a recent in vitro study, iron sucrose led to impaired phagocytic function and increased polymorphonuclear leukocyte apoptosis. [26] However, the current evidence in clinical practice cannot determine whether iron supplementation increases the risk of infection or worsens outcomes due to infection. [27, 28] There is increasing data to suggest that infective and adverseevent risks may be related to the intensity and frequency of IV iron dosing. [29-31] Brookhart et al. [32] studied iron dosing patterns in a retrospective cohort of 117.050 prevalent hemodialysis (HD) patients and found that administration of large boluses of IV iron for repleting iron deficiency was associated with increased infection-related hospitalization or death compared with smaller doses of IV iron maintenance therapy. The risk of infection-related hospitalization was increased further in patients who experienced infections within the past month. Similarly, the DOPPS study showed a trend towards an increase infection related mortality in prevalent HD patients treated with $>300 \mathrm{mg}$ of IV iron. [33] A recent meta-analysis of randomized controlled trials evaluating IV iron use (often administered as frequent boluses) in patients with varying infective risk profiles found IV iron to be associated with a $30 \%$ greater risk of infection compared with oral or no iron therapy. [34] Conversely, a prospective observational study of 985 patients failed to demonstrate a relationship between infection and serum ferritin or IV iron dosing. [35] In our study, large IV iron boluses were an effective treatment for anemia that was caused by different etiologies, because hemoglobin increased at 2 or 3 points at the end of the treatment and we found any relationship between IV iron administration and infection, which was similar to the previously published data. Furthermore, in our study, transferrin saturation increased to $23.5 \%$, thereby leaving little to no unbound iron available for bacterial utilization, as shown in previous in vitro studies. [10] For example, in the K. pneumonia case, bacterial suppression occurred throughout its incubation until the transferrin saturation exceeded $60 \%$, at which time bacterial growth occurred. [19] Another in vitro study showed that the inhibition of Staphylococcus epidermidis growth was lost once the transferrin saturation exceeded 80\%. [18] Subsequent studies had ambiguous results that left the relationship somewhat unclear. $[36,37]$ In a retrospective observational cohort study of 23.000 adult patients on HD hospitalized for bacterial infection, Ishida et al. found no association between iron treatment and readmission for infection. [38] Feldman's group, in a re-analysis of their HD cohort using multivariable analysis, showed no statistically significant association between any level of iron administration and mortality. [39] Moreover, a French multicenter prospective study regarding bacteremia risk factors in HD patients (EPIBACDIAL) did not find any correlation between parenteral iron or ferritin and bacteremia. [40] Furthermore, in the Anker study, quality of life and functional status improved in heart failure (a relatively high-risk group) without an increase in infections. [41]

Our study had limitations due to its retrospective nature. However, of the patients who were analyzed, we found a $12.8 \%$ infections after the beginning of iron therapy. Notably, an important difference in our study compared with the previously published studies was that we did not find any association between IV iron treatment and intracellular pathogens. 
In conclusion, based on our study, iron treatment is safe and it does not associate with the development of new infections. However, the infection relationship is not clear and many studies are treating to investigate the different mechanisms regarding this issue. It is important to verify this result with other studies to improve the care and future treatment of patients because iron treatment is frequently used worldside. This knowledge can improve anemia management and avoid side effects. Thus, large multi-centered randomized controlled trials designed to evaluate both the short- and long-term safety of different IV iron dosing regimens are still required to determine the optimal iron therapy.

\section{Conflict of interests}

No conflict of interest exists and all authors had full access to all the data used in the study and take full responsibility for the decision to submit the manuscript for publication.

\section{References}

1. Guerinot ML (1994) Microbial iron transport. Annu Rev Microbiol 48: 743-772. [crossref]

2. Khan FA, Fisher MA, Khakoo RA (2007) Association of hemochromatosis with infectious diseases: expanding spectrum. Int J Infect Dis 11: 482-487. [crossref]

3. Wander K, Shell-Duncan B, McDade TW (2009) Evaluation of iron deficiency as a nutritional adaptation to infectious disease: an evolutionary medicine perspective. Am J Hum Biol 21: 172-179.

4. Byrd TF, Horwitz MA (1989) Interferon gamma-activated human monocytes downregulate transferrin receptors and inhibit the intracellular multiplication of Legionella pneumophila by limiting the availability of iron. J Clin Invest 83: 1457-1465.

5. Clemens DL, Horwitz MA (1996) The Mycobacterium tuberculosis phagosome interacts with early endosomes and is accessible to exogenously administered transferrin. J Exp Med 184: 1349-1355.

6. Murray MJ, Murray AB, Murray MB, Murray CJ (1978) The adverse effect of iron repletion on the course of certain infections. Br Med J 2: 1113-1115. [crossref]

7. Sazawal S, Black RE, Ramsan M, Chwaya HM, Stoltzfus RJ, Dutta A, et al. (2006) Effects of routine prophylactic supplementation with iron and folic acid on admission to hospital and mortality in preschool children in a high malaria transmission setting: community-based, randomised, placebo-controlled trial. Lancet 367: 133-1343.

8. Clinical practice guidelines and clinical practice recommendations for anemia in chronic kidney Disease in adults (2006) Am J Kid Dis 47 (5 suppl3): S16-S85.

9. Zager RA (2006) Parenteral iron compounds: potent oxidants but mainstays of anemia management in chronic renal disease. Clin J Am Soc Nephrol 1 Suppl 1: S24-31. [crossref]

10. Parkkinen J, von Bonsdorff L, Peltonen S, Grönhagen-Riska C, Rosenlöf K. (2000) Catalytically active iron and bacterial growth in serum of haemodialysis patients after i.v. iron-saccharate administration. Nephrol Dial Transplant 15: 1827-1834.

11. Slotki I (2005) Intravenous iron supplementation in the anaemia of renal and cardiac failure--a double-edged sword? Nephrol Dial Transplant 20 Suppl 7: vii1623. [crossref]

12. Bullen JJ, Rogers HJ, Spalding PB, Ward CG (2005) Iron and infection: the heart of the matter. FEMS Immunol Med Microbiol 43: 325-330. [crossref]

13. Fishbane S (1999) Review of issues relating to iron and infection. Am J Kidney Dis 34: S47-52. [crossref]

14. Weinberg ED (1984) Iron withholding: a defense against infection and neoplasia. Physiol Rev 64: 65-102. [crossref]

15. Armitage AE, Eddowes LA, Gileadi U, Cole S, Spottiswoode N, et al. (2011) Hepcidin regulation by innate immune and infectious stimuli. Blood 118: 41294139. [crossref]

16. Boelaert JR, Vandecasteele SJ, Appelberg R, Gordeuk VR (2007) The effect of the host's iron status on tuberculosis. J Infect Dis 195: 1745-1753. [crossref]

17. McDermid JM, Jaye A, Schim van der Loeff MF, Todd J, Bates C, Austin S, et al. (2007) Elevated iron status strongly predicts mortality in West African adults with HIV infection. J Acquir Immune Defic Syndr 46: 498-507.

18. Brewster UC, Perazella MA (2004) Intravenous iron and the risk of infection in end-stage renal disease patients. Semin Dial 17: 57-60. [crossref]

19. Cieri E (1999) Does iron cause bacterial infections in patients with end stage renal disease? ANNA J 26: 591-596. [crossref]
20. Rivera S, Nemeth E, Gabayan V, Lopez MA, Farshidi D, Ganz T (2005) Synthetic hepcidin causes rapid dose-dependent hypoferremia and is concentratedin ferroportin-containing organs. Blood 106: 2196-2199.

21. Ishida JH, Johansen KL (2014) Iron and infection in hemodialysis patients. Semin Dial 27: 26-36. [crossref]

22. Gupta A, Zhuo J, Zha J, Reddy S, Olp J, Pai A (2010) Effect of different intravenous iron preparations on lymphocyte intracellular reactive oxygen species generation and subpopulation survival. BMC Nephrol 11: 16.

23. Brewster UC (2006) Intravenous iron therapy in end-stage renal disease. Semin Dial 19: 285-290. [crossref]

24. Deicher R, Ziai F, Cohen G, Müllner M, Hörl WH (2003) High dose parenteral iron sucrose depresses neutrophil intracellular killing capacity. Kidney Int 64: 728-736.

25. van Asbeck BS, Marx JJ, Struyvenberg A, Verhoef J (1984) Functional defects in phagocytic cells from patients with iron overload. J Infect 8: 232-240. [crossref]

26. Ichii H, Masuda Y, Hassanzadeh T, Saffarian M, Gollapudi S, Vaziri ND (2012) Iron sucrose impairs phagocytic function and promotes apoptosis in polymorphonuclear leukocytes. Am J Nephrol 36: 50-57.

27. Kliger AS, Foley RN, Goldfarb DS, Goldstein SL, Johansen K, et al. (2013) KDOQI US commentary on the 2012 KDIGO Clinical Practice Guideline for Anemia in CKD. Am J Kidney Dis 62: 849-859. [crossref]

28. Shah A, Roy NB2, McKechnie S3, Doree C4, Fisher SA4, et al. (2016) Iron supplementation to treat anaemia in adult critical care patients: a systematic review and meta-analysis. Crit Care 20: 306. [crossref]

29. Feldman HI, Santanna J, Guo W, Furst H, Franklin E, et al. (2002) Iron administration and clinical outcomes in hemodialysis patients. $J$ Am Soc Nephrol 13: 734-744. [crossref]

30. Freburger JK, Ellis AR, Kshirsagar AV, Wang L, Brookhart MA (2014) Comparative short-term safety of bolus versus maintenance iron dosing in hemodialysis patients: a replication study. BMC Nephrol 15: 154.

31. Agarwal R, Kusek JW, Pappas MK (2015) A randomized trial of intravenous and oral iron in chronic kidney disease. Kidney Int 88: 905-914. [crossref]

32. Brookhart MA, Freburger JK, Ellis AR, Wang L, Winkelmayer WC, et al. (2013) Infection risk with bolus versus maintenance iron supplementation in hemodialysis patients. J Am Soc Nephrol 24: 1151-1158. [crossref]

33. Bailie GR, Larkina M, Goodkin DA, Li Y, Pisoni RL, Bieber B, et al. (2015) Data from the Dialysis Outcomes and Practice Patterns Study validate an association between high intravenous iron doses and mortality. Kidney Int 87: 162-168.

34. Litton E, Xiao J, Ho KM (2013) Safety and efficacy of intravenous iron therapy in reducing requirement for allogeneic blood transfusion: systematic review and metaanalysis of randomized clinical trials. BMJ 347: f4822.

35. Hoen B, Paul-Dauphin A, Hestin D, Kessler M (2002) Intravenous iron administration does not significantly increase the risk of bacteriemia in chronic hemodialysis patients. Clin Nephrol 57: 457-461.

36. Bansal A, Sandhu G, Gupta I, Kalahalli S, Nayak R, Zouain E, et al. (2014) Effect of aggressively driven intravenous iron therapy on infectious complications in endstage renal disease patients on maintenance hemodialysis. Am J Ther 21: 250-253.

37. Miskulin DC, Tangri N, Bandeen-Roche K, Zhou J, McDermott A, et al. (2014) Intravenous iron exposure and mortality in patients on hemodialysis. Clin J Am Soc Nephrol 9: 1930-1939. [crossref]

38. Ishida JH, Marafino BJ, McCulloch CE, Dalrymple LS, Dudley RA, et al. (2015) Receipt of Intravenous Iron and Clinical Outcomes among Hemodialysis Patients Hospitalized for Infection. Clin J Am Soc Nephrol 10: 1799-1805. [crossref]

39. Feldman HI, Joffe M, Robinson B, Knauss J, Cizman B, et al. (2004) Administration of parenteral iron and mortality among hemodialysis patients. J Am Soc Nephrol 15: 1623-1632. [crossref]

40. Hoen B, Paul-Dauphin A, Hestin D, Kessler M (1998) EPIBACDIAL: a multicenter prospective study of risk factors for bacteremia in chronic hemodialysis patients. $J$ Am Soc Nephrol 9: 869-876. [crossref]

41. Anker SD, Comin Colet J, Filippatos G, Willenheimer R, Dickstein K, et al. (2009) Ferric carboxymaltose in patients with heart failure and iron deficiency. $N$ Engl $J$ Med 361: 2436-2448. [crossref]

\section{Citation:}

Emilio Fábrega, María José García, Carmen Alonso, Manuel FernandezMiera, et al. (2017) Is intravenous iron treatment a risk for infection?. Internal Med Res Open J Volume 2(1): 1-4 